\title{
THREE DIMENSIONAL COMPLEX SHAPES ANALYSIS FROM 3D LOCAL CURVATURE MEASUREMENTS. APPLICATION TO INTERMETALLIC PARTICLES IN ALUMINIUM ALLOY 5XXX
}

\author{
Estelle Parra-Denis ${ }^{1}$, Nicolas Moulin ${ }^{2}$ AND DOMINiQue JeUlin ${ }^{1}$ \\ ${ }^{1}$ Centre de Morphologie Mathématique, Ecole Nationale Supérieure des Mines de Paris, Fontainebleau, France; \\ ${ }^{2}$ Ecole Nationale Supérieure des Mines de Saint-Etienne, Saint-Etienne, France \\ e-mail: estelle.parra@ensmp.fr,nmoulin@emse.fr, dominique.jeulin@ensmp.fr \\ (Accepted October 8, 2007)
}

\begin{abstract}
The studied material is a $5 \mathrm{xxx}$ aluminium alloys containing 2 types of intermetallic particles : $A l_{x}(F e, M n)$ and $\mathrm{Mg}_{2} \mathrm{Si}$. It is usually used in car industry as reinforcement pieces or in packaging industry, such as bottle liquid box lid. Scanning electronic microscope coupled with EDX analysis shows complex shapes of intermetallic particles. The particle shape is obtained during the solidification of alloys. Particles fill vacant spaces between aluminium grains. Therefore final sheet properties depend on intermetallic particles shapes and notably on the matrix-particle interface properties. The goal of the present study is to classify intermetallic particles versus their shapes using local curvature information. The aluminium alloys sample is observed by $\mathrm{X}$ ray micro tomography performed at the ESRF. Three dimensional images are segmented, and intermetallic particles are identified in a data base. Each particle is stored as a set of voxels. The surface of each particle is meshed by a marching cubes triangular meshing with the software Amira $\odot$. A simplification of the surface is performed by an algorithm contracting the edges. Finally, principal curvatures: $k_{\min }$ and $k_{\max }$ are estimated by Amira@ on each facet centre of the mesh. From the full intermetallic population, the bivariate distribution of $k_{\min }$ and $k_{\max }$ is estimated. The obtained graph $k_{\min }-k_{\max }$ shows geometrical properties of interface portions of the surface of particles. A factorial correspondence analysis is performed to summarize the information on all intermetallic particles. In the obtained subspace, particles are classified into five shape families, in relation with their interface geometrical properties.
\end{abstract}

Keywords: 3D, classification, complex shape, local curvature, statistical analysis.

\section{INTRODUCTION}

The study of the microstructure of materials allows to predict its mechanical properties, and to develop a product with desired properties.

$\mathrm{X}$ ray micro tomography performed at the ESRF (European Synchrotron Radiation Facility) provides the acquisition of three dimensional images of materials in a non destructive way with a high resolution (up to $0.7 \mu \mathrm{m}$ in the present case). Image analysis is then fundamental to extract information within scanned images, and to quantitatively characterize the micro structure of materials from three dimensional measurements.

In the case of metallic materials, as aluminium alloys $5 \mathrm{xxx}$, the microstructure contains intermetallic particles showing 3D complex shapes. Their analysis requires characterization method suited to the complex nature of information. Many 3D shape analysis methods exist in the literature. Nevertheless, in most cases they are used for simple 3D shape analysis, such as for instance star shapes are described with the help of Fourier descriptors (Gardner et al., 2003; Hobolth, 2003). For more complex shapes, parametric studies have been efficiently applied (Parra-Denis et al., 2007; Parra-Denis, 2007).

In this paper, we propose an alternate methodology to the parametric one for the three dimensional complex shapes analysis. This analysis is based on the study of the principal local curvature $k_{\min }$ and $k_{\max }$ at the surface of objects. This methodology follows the ideas of Alkemper and Voorhees (2001); Voorhees et al. (2002) to study the growth of aluminium grain dendrites. In the present study, we use the bivariate distribution of $k_{\min }$ and $k_{\max }$ to analyse the three dimensional complex shapes of intermetallic particles.

The paper is organized as follows: in the first part, we provide the method used to obtain the local curvature's information. Firstly, the definition of principal curvatures on an object surface is given. Secondly, the object's meshing method used in the present study is detailed. Lastly, we give parameters of the meshing for intermetallic particles contained in aluminium alloy $5 \times x x$. In the second part, we present the bivariate distribution of $k_{\min }$ and $k_{\max }$. Firstly, we give interpretation of the graph. Then, an example on a typical intermetallic particle illustrates the information of the graph. In the third part, the 
statistical analysis of the results on an intermetallic particles population is presented. Its implementation is first given. Then the results obtained are presented. Finally, a classification depending on intermetallic particle shapes is performed. In the last part, we conclude with presenting the advantages and the limits of the proposed method, and present some possible projects for future works.

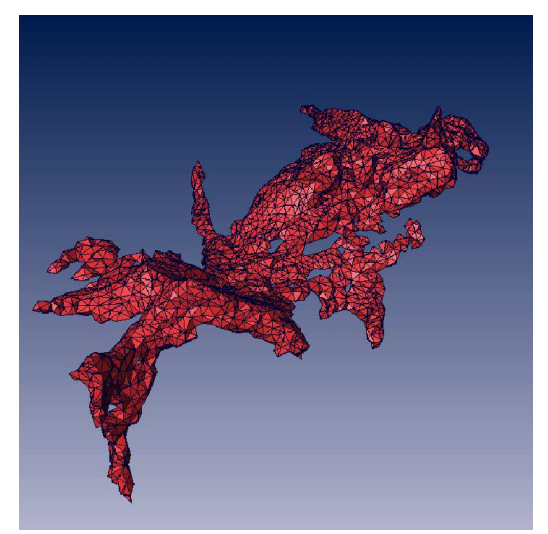

Fig. 1. Example of intermetallic particles contained in AA 5xxx meshed by Amira. The facets number of the meshing is equal to 8044.

\section{MEASUREMENTS OF LOCAL 3D CURVATURE}

In image analysis, the study of the curvature is used to measure the total curvature integral which presents topological features, and the mean curvature integral which presents metrical features. They are estimated by stereological methods (Ohser and Mücklich, 2000). The study of the curvature is also currently used in computer generated images. In these studies, the discrete surface of objects is characterized by the local curvature measurement (Besl and Jain, 1986; Stokely and $\mathrm{Wu}, 1992)$. These methods are based on a triangular meshing of the surface (Polthier and Rossman, 2002).

In metalworking industry, the studies performed by Mendoza, Alkemper and Voorhees (Alkemper and Voorhees, 2001; Voorhees et al., 2002) on the local curvature histograms ensure to follow the evolution of aluminium dendrites during the solidification process. An application of these studies to intermetallic particle shapes contained in aluminium alloys seems to be appropriate.

In this section, we present the measurement of local curvature to characterize three dimensional complex shapes. The definition of surface curvature in the three dimensional space is first defined. Then, its implementation is presented. Lastly, parameters of the meshing for intermetallic particles contained in aluminium alloy are given.

\section{THE CURVATURE OF A SURFACE}

Let $S$ be a smooth surface in the Euclidean space $\mathbb{R}^{3}$, and $x$ be a point of $S$. The normal vector $n$ is defined at $x$ on the surface $S$. Every plane $P$ containing $n$ intersects $S$ with a curve $C$. For each plane $P_{i}$, the radius of curvature $r_{i}$ of $C_{i}$ at $x$ is given. The minimum curvature radius $R_{\min }$ and the maximum one $R_{\max }$ are obtained for a set of two orthogonal planes. They define the maximum curvature $k_{\max }$ (reciprocal of the radius) and the minimum curvature $k_{\min }$, called principal curvatures.

\section{IMPLEMENTATION}

The surface meshing of an object is usually used in materials field, to carry out a mechanical analysis of an object by finite elements calculation, directly from three dimensional images. The choice of the technique to mesh the surface is a difficult problem (Frey and George, 1999).

In the present study, the local curvature measurement is performed with Amira $\bigcirc$ software. The principal curvatures of intermetallic particles are measured on the mesh produced by Amira $\odot$.

The geometry of aluminium alloys containing particles is complex (Fig. 1). It is far from simple geometries and the use of regular meshing of points is not possible; then the method used to mesh particles is a frontal non structuring method.

Frontal methods mesh the field by successive iterations, propagating a front inside the surface (starting on the surrounding area of the field), until its complete covering.

The non structuring meshing method used is based on the Marching Cube algorithm (Lorensen and Cline, 1987). From the 3D image, the surface of the object is digitized into triangles to mesh. Then from the discrete surface, the volume is meshed into linear tetrahedrons by using a frontal method implemented into Amira $\odot$ software. This method allows us to vary the length of elements of the model without creating discontinuities. The number of triangular elements needed to cover the surface depends on its complexity. It is optimized to obtain a good definition of the surface with a low number of degrees of freedom.

After meshing, a simplification algorithm of the surface by collapsing edges is applied (Hoppe et al., 1993). This algorithm consists in merging the two 
extremities of edges into an apex, with a collapsing criterion based on the minimization of an energy function (Tables 1 and 2).

\section{MEASURES PARAMETERS USED}

The measure of the curvature in Amira $\odot$ software is based on the meshing obtained by the Marching Cubes algorithm. Thus the principal curvatures are estimated either for each triangle of the surface, or for each point of the meshing. The algorithm works by a local fit of the surface according to a quadratic form. The eigenvalues and eigenvectors of the quadratic form correspond to the principal curvature values and to the directions of principal curvature.

Then a smoothing is made, depending on two parameters:

- The number of neighbours: it determines which triangles and which points are considered to be neighbours of a given triangle and of a given point. If the value of this input is one, then only triangles sharing an edge with a given triangle, and only points directly connected to a given point are considered.

- The number of iterations: it determines how many times the initial curvature values computed for a triangle or for a point are being averaged with the curvature values of direct neighbour triangles or points. This parameter only applies to the scalar curvature values, not to the directional curvature.

A code written in Amira $\odot$ software automatically generates the measurement of the curvature on an images set of raw type. For a sample of aluminium alloys with dimensions $(1 \times 1 \times 4) \mathrm{mm}^{3}$, each intermetallic particle corresponds to a single binary image. Typically there are among 4.000 particles in a sample. Each particle is then meshed. Amira $\bigcirc$ gives, for each facet of the meshed object, the coordinates of the three points which compose the triangle, and the values of the principal curvatures. A file containing each facet of the meshing its surface and its principal curvatures values is created. A preliminary study on different usual shapes with varying length was being performed, in order to validate the method of estimation. We have tested different shapes: cylinder (with different orientations), sphere, torus, and ellipsoid. For each tested shape, we introduce variations of all the parameters: simplification of the meshing varying between $0 ; 1,4 ; 2,8$, number of neighbours varying between $1 ; 6 ; 16$. Finally the retained parameters for further measurements are a simplification of the meshing equal to 1.4 , a number of layers equal to 6 , and a number of average equal to zero (Fig. 1 and Table 1).
The time needed to numerically compute the different curvatures on a data base of 4921 images of particles is $2 \mathrm{hrs} 21 \mathrm{~min}$ (on a AMD Athlon 2600+ with 2 GB of RAM).

\section{THE $k_{\min }-k_{\max }$ GRAPH}

It is possible to quantify the shape of the objectmatrix interface by looking at the distribution of the principal curvatures $\left(k_{\min }-k_{\max }\right)$. The papers written by Alkemper, Voorhes and Mendoza present the use of the obtained graph to characterize the growth of dendrites during the solidification process of copper aluminium alloys (Alkemper and Voorhees, 2001; Voorhees et al., 2002).

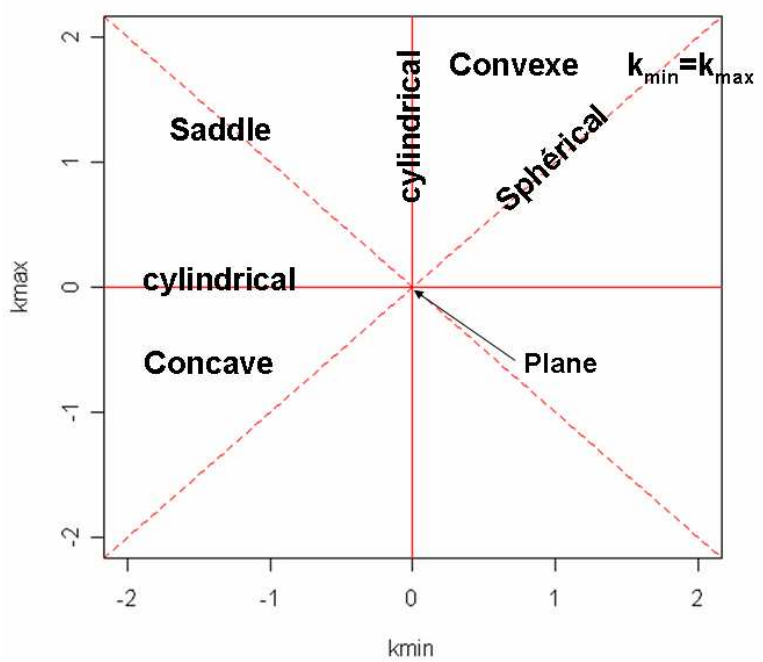

Fig. 2. $k_{\min }-k_{\max }$ graph with the different types of portion intrefaces corresponding region.

\section{GEOMETRICAL PROPERTIES}

The $\left(k_{\min }-k_{\max }\right)$ graph presented in the reference article of Besl and Jain (1986) presents the geometrical properties of interface portions (Fig. 2). The shape of the interface can be determined from the values of $k_{\text {min }}$ and $k_{\max }$. Examples of different geometrical interface portion types are illustrated in Fig. 3. We present the striking properties of this graph (see Fig. 2):

- All the points of the surface are located on the left of the line corresponding to $k_{\min }=k_{\max }$. All the interface portions belonging to this line have a spherical shape.

- The planar interface portions are located at the origin of the graph, where $k_{\min }=k_{\max }=0$. 
Table 1. Values of the surface and volume measure on different shapes with different values of simplification meshing.

\begin{tabular}{llrrrr} 
& & Cylinder 3D & 3D turned cylinder & Oblate spheroid & Torus \\
\hline \multirow{2}{*}{ Contract edges 2,8 } & volume & 328274 & 327698 & 989383 & 1168340 \\
\cline { 2 - 6 } & surface & 26928,6 & 26929,2 & 539323 & 77845 \\
\hline Contract edges 2,0 & volume & 328520 & 328024 & 990032 & 1169135 \\
\cline { 2 - 6 } & surface & 26960,9 & 27142,1 & 54099 & 78073 \\
\hline Contract edges 1,4 & volume & 328691 & 327890 & 990394 & 1169416 \\
\cline { 2 - 6 } & surface & 27572,4 & 27829,2 & 55620 & 79970 \\
\hline Contract edges 0,0 & volume & 328918 & 328000 & 990371 & 1169213 \\
\cline { 2 - 6 } & surface & 28227,7 & 29818,8 & 58448 & 83872 \\
\hline
\end{tabular}

Table 2. Meshing number elements in relation to the simplification of the meshing applied and to the shapes of the studied object.

\begin{tabular}{llrrrr} 
& & Cylinder 3D & 3D turned cylinder & Oblate spheroid & Torus \\
\hline Contract edges 2.8 & points & 878 & 821 & 1766 & 2464 \\
\cline { 2 - 6 } & faces & 1752 & 1638 & 3528 & 4928 \\
\cline { 2 - 6 } & edges & 2628 & 2457 & 5292 & 7392 \\
\hline Contract edges 2.0 & points & 1734 & 1642 & 3476 & 4986 \\
\cline { 2 - 6 } & faces & 3462 & 3278 & 6948 & 9972 \\
\cline { 2 - 6 } & edges & 5193 & 4917 & 10422 & 14958 \\
\hline Contract edges 1.4 & points & 3994 & 3558 & 6805 & 9952 \\
\cline { 2 - 6 } & faces & 7984 & 7112 & 13602 & 19886 \\
\cline { 2 - 6 } & edges & 11976 & 10668 & 20403 & 29829 \\
\hline Contract edges 0,0 & points & 32678 & 39816 & 76534 & 110656 \\
\cline { 2 - 6 } & faces & 6552 & 79628 & 153064 & 221312 \\
\cline { 2 - 6 } & edges & 98028 & 119442 & 229596 & 331968 \\
\hline
\end{tabular}

Table 3. Clustering of intermetallic particles into 5 shape families according to their matrix-particle interface properties.

\begin{tabular}{lll}
\hline Type 1 & Saddle IP +++ & Branched particles \\
\hline Type 2 & $\begin{array}{l}\text { Flat IP }+++ \text { Ridge } \\
\text { IP. Valley IP }+\end{array}$ & $\begin{array}{l}\text { Flat Particles with } \\
\text { an important aspect } \\
\text { ratio }\end{array}$ \\
\hline Type 3 & $\begin{array}{l}\text { Flat IP }+++ \text { Concave } \\
(120)\end{array}$ & $\begin{array}{l}\text { Flat particles with } \\
\text { concavity }\end{array}$ \\
\hline Type 4 & Ridge IP +++ Flat IP & Sharped particles \\
\hline Type 5 & Spherical IP +++ & $\begin{array}{l}\text { Particles with a } \\
\text { rounded interface }\end{array}$ \\
\hline
\end{tabular}


- The cylindrical interface portions have $k_{\min }=0$ or $k_{\max }=0$; if $k_{\min }=0$ the portion has a valley shape, if $\mathrm{kmax}=0$ it has a ridge shape.

- The hyperbolic interface portions or saddle shape have one positive and one negative curvature.

- If the two principal curvature values are positive, the interface portion is concave.

- If the two principal curvature values are negative, the interface portion is convex.
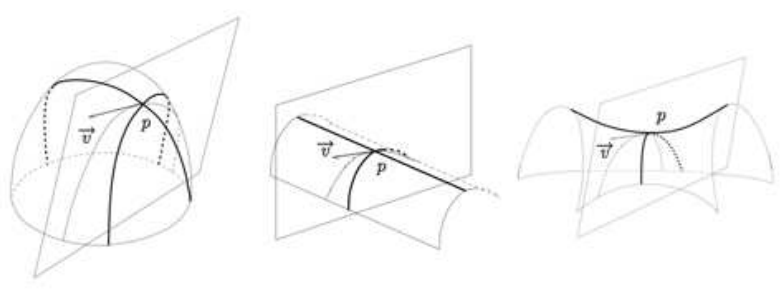

Fig. 3. Geometry of typical interface at the point $p$.

\section{THE BIVARIATE DISTRIBUTION \\ OF $\left(k_{\min }-k_{\max }\right)$}

On the $\left(k_{\min }-k_{\max }\right)$ graph each point represents a portion of the object interface. In the case of intermetallic particles the obtained meshing is defined by a large number of facets, from one thousand up to at least ten thousands (Table 2). A two-dimensional histogram can then be used to correctly exploit the available information.

From the curvature result files we have plotted the $\left(k_{\min }-k_{\max }\right)$ graph (see Fig. 4) and the empirical bivariate distribution of $\left(k_{\min }-k_{\max }\right)$ (see Fig. 5) for each intermetallic particles contained in the data base. The bivariate distributions of $\left(k_{\min }-k_{\max }\right)$ are weighted, each facet being counted according to the amount of the object surface it represents (see Fig. 5).

\section{RESULTS}

The bivariate distribution of $\left(k_{\min }-k_{\max }\right)$ of the particle shown in Fig. 1 is given in Fig. 5. It presents a significant concentration of interface located: on the line $k_{\max }=0$, on the line $k_{\min }=0$, into the saddle shape interface portion area and in a peak area centred on zero. It suggests that the particle has an important number of cylindrical interface portions with ridge type, and also a large part of planar interface portions. The particle is then made of planar area and of branches with intersections.

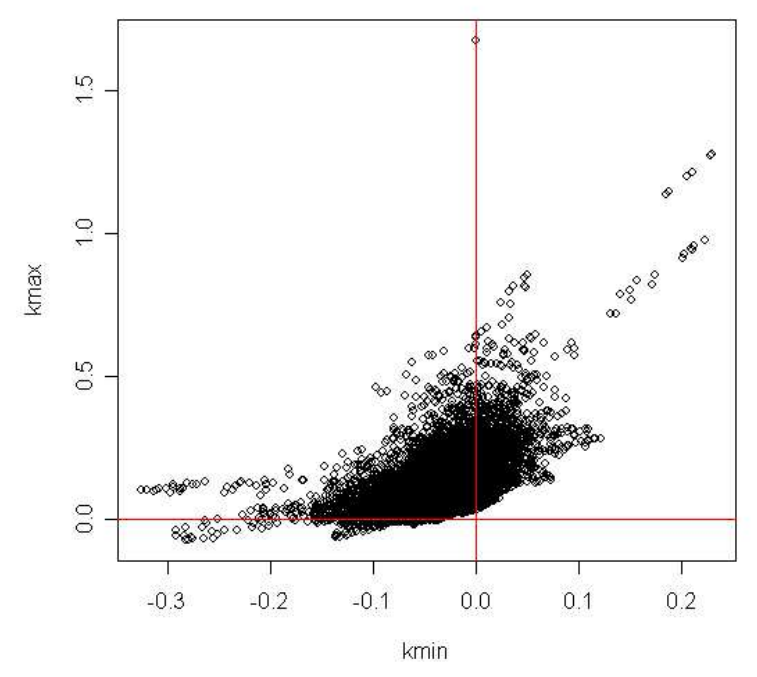

Fig. 4. $\left(k_{\min }-k_{\max }\right)$ graph of particle illustrated in Fig. 1.

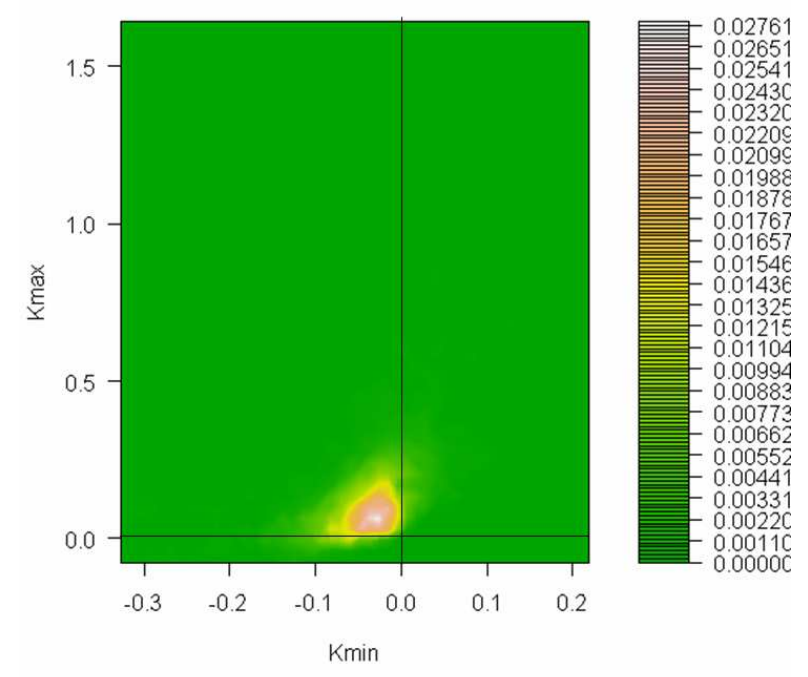

Fig. 5. Bivariate distribution $\left(k_{\min }-k_{\max }\right)$ of the particle illustrated in Fig. 1.

\section{STATISTICAL ANALYSIS OF THE BIVARIATE DISTRIBUTION ON A POPULATION OF INTERMETALLIC PARTICLES}

\section{PRINCIPLE}

In the case of an important population of complex shape particles, we have to perform a statistical analysis. The goal of the study is to describe shapes of populations of objects by using an adapted representation space. For each intermetallic particle 
extracted from the sample of aluminium alloy 5xxx, we estimate the bivariable distribution $\left(k_{\min }-k_{\max }\right)$. The ranges of each graph are kept constant, in order to be able to compare the graphs of the particles.

A factor correspondence analysis of the bivariate distribution is performed, to compare the distributions in a space provided with the Khi 2 distance (Benzécri, 1980). In this space, it is possible to classify particles by using a Hierarchical Cluster Analysis (HCA). The Ward's algorithm is used (Ward, 1963). Initially, each particle is assigned to its own cluster. Then the algorithm proceeds iteratively. At each stage, it joins the two most similar clusters, by using as distance between two clusters, the loss of inertia generated by merging two classes. It is iterated until a single cluster is remaining.

The HCA leads to a hierarchical clustering into n clusters, represented by tree-like structures, called dendrograms (Fig. 6). A dendrogram shows the $n-1$ steps of the clustering process leading to a single cluster. A truncation at a given level of the dendrogram, gives a partition of the population. The goal of the HCA is to globally indicate the presence of clusters. If at a level of the dendrogram the intra clusters inertia sharply increases, we consider that the classification of particles is pertinent.

\section{RESULTS}

Fig. 6 represents the dendrogram obtained by the Ward's method on the intermetallic particles population of the $5 \mathrm{xxx}$ aluminium alloy. The choice of the number of clusters by cutting the tree is obtained visually. The levels of aggregation are observed to vary between 0 and 160 in Fig. 6. The last knots, visualized by red dot lines in Fig. 6, represent the most significant aggregation jumps. We can expect a correct partition of the particles population by cutting the tree between the knots' values 35 and 45 . Indeed, a cut at this level of the dendrogram represents an important aggregation jump, and gives five clusters. The five identified particle clusters have geometrical properties summarised in Table 3. Figs. 7 to 11 illustrate each particle family contained in the aluminium alloy.

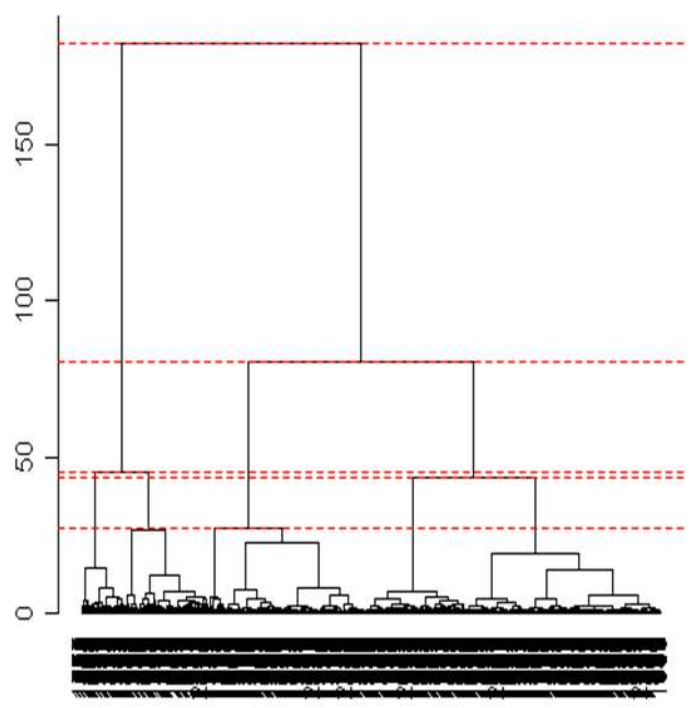

Fig. 6. Dendrogram of the intermetallic particles' contained into AA5xxx (HCA obtained by Ward's method).

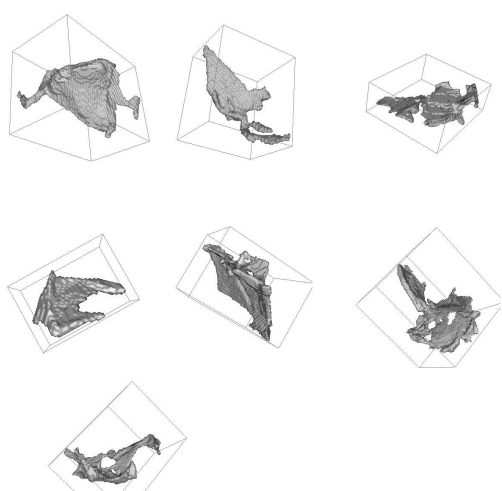

Fig. 7. Examples of intermetallic particles contained into type 1 .

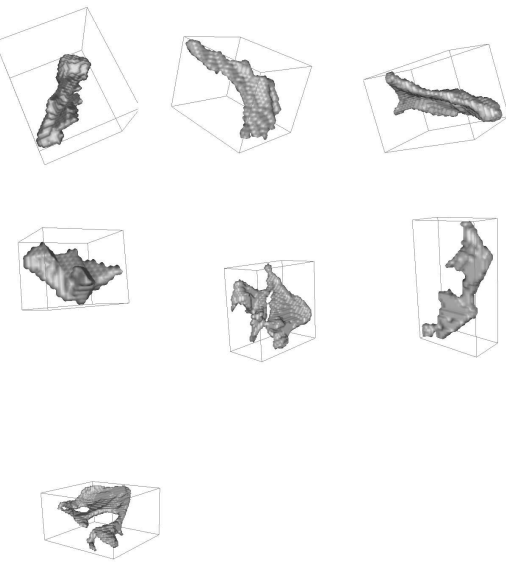

Fig. 8. Examples of intermetallic particles contained into type 2. 


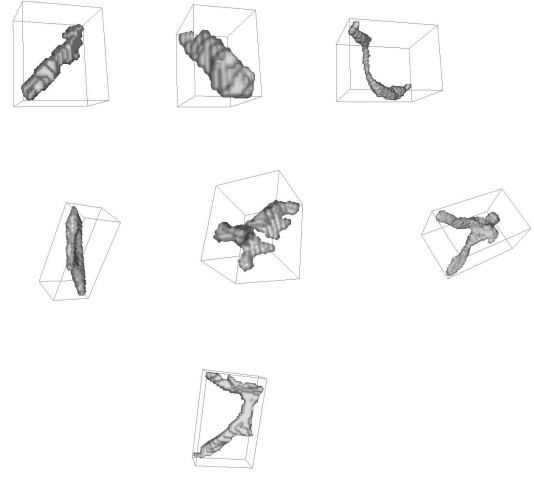

Fig. 9. Examples of intermetallic particles contained into type 3.

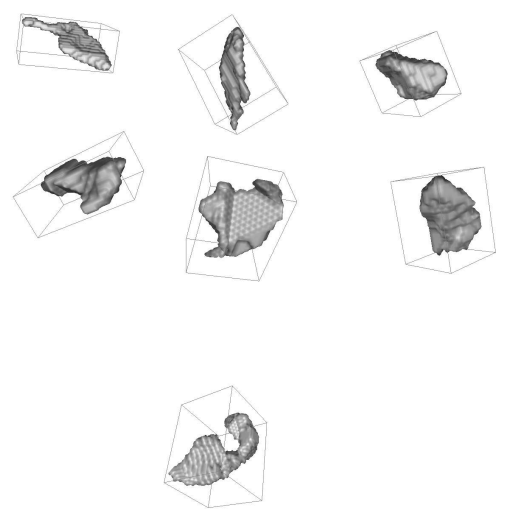

Fig. 10. Examples of intermetallic particles contained into type 4.

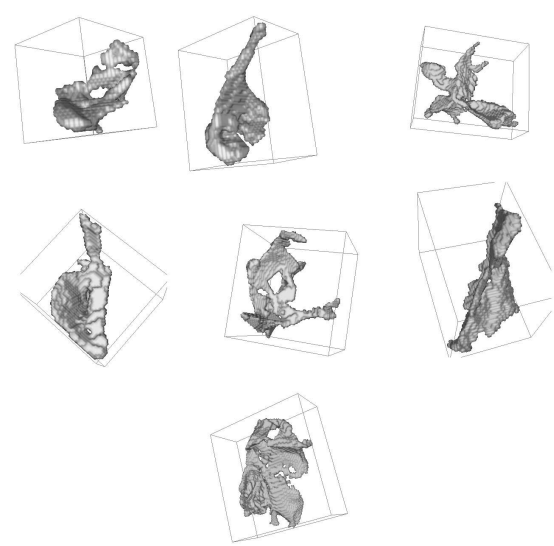

Fig. 11. Examples of intermetallic particles contained into type 5 .

\section{CONCLUSION}

The characterization of the three-dimensional complex shape of objects by means of curvature information is efficient. This characterization method is original; it gives complex information about the nature of the interface between three dimensional objects and the material in their surrounding area. A relevant classification of objects with complex shape according to the statistics of their geometrical properties at the interface (namely the principal curvature) is possible. The curvature gives interesting information for mechanical studies on intermetallic particles; in particular for futures studies on the break up of intermetallic particles during industrial process according to their families.

\section{REFERENCES}

Alkemper J, Voorhees PW (2001). Three dimensionnal characterization of dendritic microstructures. Acta Mater 49:897-902.

Benzécri JP (1980). La Pratique de L'analyse Des Données, vol. II. Bordas, Paris, Bruxelles, Montréal: Dunod, 2nd ed.

Besl PJ, Jain RC (1986). Invariant surface characteristics for 3D object recognition in range images. Comput Vision Graph 33:33-80.

Frey PJ, George PL (1999). Maillage: Applications aux éléments finis. Science Publications.

Gardner RJ, Jensen EBV, Volcic A (2003). Geometric tomography and local stereology. Adv Appl Math 30:397-423.

Hobolth A (2003). The spherical deformation model. Biostatistics 4:583-95.

Hoppe H, DeRose T, Duchamp T, McDonald J, Stuetzle W (1993). Mesh optimization. In: Kajiya JT, ed., Proceedings of ACM SIGGRAPH 1993.

Lorensen WE, Cline HE (1987). Marching cubes: A high resolution 3D surface construction algorithm. In: SIGGRAPH '87: Proceedings of the 14th annual conference on Computer graphics and interactive techniques. New York, NY, USA: ACM Press.

Ohser J, Mücklich F (2000). Statistical Analysis of Microstructures in Materials Science. John Wiley \& Sons.

Parra-Denis E (2007). Analyse morphologique 3D de particules de forme complexe: application aux intermétalliques dans les alliages d'aluminium. Ph.D. thesis, Université Jean Monnet de Saint-Etienne.

Parra-Denis E, Barat C, Ducottet C, Jeulin D (2007). 3D complex shape characterization by statistical 
analysis: Application to aluminium alloys. Accepted for publication in Materials Characterization .

Polthier K, Rossman W (2002). Index of discrete constant mean curvature surfaces. J Reine Angew Math 549:4777.

Stokely EM, Wu SY (1992). Surface parametrization and curvature measurement of arbitrary 3-d objects: Five practical methods. IEEE Trans Pattern Anal 14:833-40.

Voorhees PW, Alkemper J, Mendoza R (2002). Morphological evolution of dendritic microstructures. Adv Eng Mater 4:481-9.

Ward JH (1963). Hierarchical grouping to optimize an objective function. J Am Stat Assoc 58:234-44. 